\title{
REPASSES DO SUS X CUSTOS DE TOMOGRAFIAS COMPUTADORIZADAS EM UMA CLÍNICA DE RADIOLOGIA NO ALTO OESTE POTIGUAR
}

\section{TRANSFERS FROM SUS X COST OF COMPUTERIZED TOMOGRAPHIES IN A RADIOLOGY CLINIC OF POTIGUAR HIGH WEST}

\author{
Francisco Jean Carlos de Souza Sampaio \\ E-mail: sampaiojean@yahoo.com.br \\ Manoela Karem Gonçalves de Lima Pereira \\ E-mail: manoelakaremg@,gmail.com
}

\section{RESUMO}

Este artigo tem como objetivo principal averiguar a viabilidade em ser prestador do SUS, estabelecendo comparações entre os custos totais e o rapasse do SUS para cada um dos procedimentos ofertados. Para isto, utilizou-se do método Activity Based Costing (ABC), neste intento, dentro do período de apuração dos dados correspondente ao primeiro semestre do ano de 2019, foram analisados os processos de atendimento e de realização dos procedimentos, os setores e as atividades neles desenvolvidas e consequentemente os recursos utilizados. Os dados foram coletados através da pesquisa de campo, da pesquisa documental e de uma entrevista aberta junto ao supervisor técnico em radiologia. Os resultados obtidos através do custeio $\mathrm{ABC}$, compreendem à realização de 3.069 procedimentos, dos quais 1.520 foram realizados por meio do convênio SUS, representando uma margem de $49,5 \%$ da demanda total realizada pela clínica em análise. Entretanto, ao correlacionar os custos aferidos a cada tipo de procedimento às tabelas de valores SUS obtém-se déficit, em todos os procedimentos ofertados. Evidenciando com isto, a inviabilidade em ser prestador de serviços SUS e a necessidade de reaver os valores contratuais dos procedimentos estudados.

Palavras-chave: Administração de Custos; Tomografia Computadorizada; Custeio por Atividades.

\begin{abstract}
The main objective of this paper is to investigate the feasibility of being a SUS provider, establishing comparisons between total costs and financial transfers from SUS for each of the procedures offered. To accomplish this objective, Activity Based Costing method (ABC) was used considering costs calculations regarding the period corresponding to the first semester of 2019, considering the processes of attendance and performance of procedures, as well as the sectors and activities developed therein and consequently the resources used. Data was collected through field research, documentary research and an open interview with the technical supervisor in radiology. The results obtained through ABC costing comprise the accomplishment of 3,069 procedures, of which 1,520 were performed through the SUS agreement, representing a margin of $49.5 \%$ of the total demand made by the clinic under analysis. However, by correlating the costs to each type of procedure to the SUS tables of values, we obtain a deficit in all the procedures offered. It has been stablished the
\end{abstract}


impossibility of being a SUS service provider and the need to recover the contractual values of the studied procedures.

Keywords: Cost Management; Computed Tomography; Activity Based Costing.

\section{INTRODUÇÃO}

Ao processo de desenvolvimento socioeconômico do Brasil, consideradas as especificidades regionais, subjazem inúmeros desafios, dentre os quais se destaca à necessidade de instauração de uma dinâmica organizacional das empresas que lhes assegure saúde financeira no universo competitivo do mercado, cujas demandas apontam a absorção dos incrementos advindos das revoluções tecnológica e científica, bem como dos padrões empresariais adotados na contemporaneidade.

Nesse contexto de desafios, situam-se as microempresas que vivenciam fortes e diversos dilemas para sobreviverem por tempo prolongado no mercado brasileiro, valendo, aqui, registrar que há um grande número de empresas que sobrevivem, apenas até cinco anos, conforme aponta dados obtidos pelo SEBRAE, em estudos publicados no ano de 2016.

Um dos aspectos que podem ocasionar a falência das empresas pode ser a ausência de uma gestão financeira adequada às empresas. A contabilidade de custos pode contribuir, de forma enriquecedora, para a saúde financeira em clínicas e hospitais, através dos dados que serão fornecidos, que poderão ser utilizados nos mais diferentes níveis e situações, como por exemplo, ajudam na formação de preços, na mensuração dos custos e das atividades ocorridas. Nessa perspectiva, infere-se que a contabilidade de custos influencia, diretamente, na tomada de decisões, proporcionando aos gestores a compreensão necessária mediante o surgimento de necessidades e oportunidades.

O diagnóstico organizacional teve como lócus uma clínica particular situada no município de Pau dos Ferros - RN, localizada no Alto-Oeste Potiguar, a qual presta serviços do Sistema Único de Saúde - SUS, na realização de tomografias computadorizadas, o que impulsionou o desenvolvimento de uma investigação de natureza científica na área, na medida em que enfatizou a ausência de conhecimento sobre as atividades e seus custos utilizados na realização dos serviços de tomografias oferecidos pela referida clínica.

Conforme Amaro Júnior e Yamashita (2001, p. 01):

A Tomografia computadorizada, que se baseia em raios-X, foi utilizada para aplicações clínicas ainda no início da década de 70, uma vez que torna possível examinar o encéfalo e, com maior clareza, os limites do sistema ventricular e as partes ósseas do crânio. $\mathrm{O}$ aparelho consiste em uma fonte de raios-X que é acionada ao mesmo tempo em que realiza um movimento circular ao redor da cabeça do paciente, emitindo um feixe de raios-X em forma de leque. No lado oposto a essa fonte, está localizada uma série de detectores que transformam a radiação em um sinal elétrico que é convertido em imagem digital. Dessa forma, as imagens correspondem a secções ("fatias") do crânio. A intensidade (brilho) reflete a absorção dos raios-X e pode ser medida em uma escala (unidades Hounsfield).

Diante da discussão sobre os serviços de tomografia computadorizada, a pesquisa trata de uma inquietação e curiosidade científica que visam a identificar os custos operacionais na realização desses serviços, justificando a escolha temática da pesquisa, que se dá pela exteriorização dos custos operacionais dispostos à realização de tomografias computadorizadas na referida clínica, sob a ótica de averiguar a viabilidade em ser prestador 
do SUS, estabelecendo comparações entre os custos totais e o rapasse do SUS para cada um dos procedimentos ofertados.

Nesse intento, busca-se a construção de um levantamento dos custos operacionais e administrativos, através do rateio desses custos utilizando o método ABC (Activity Based Costing), calcularem quais são os custos de cada tipo de tomografia realizada e compará-los ao valor final do exame com o valor pago pelo SUS, para constatar se os valores repassados pelo SUS cobrem os custos operacionais dos procedimentos de tomografias computadorizadas, relacionando, inclusive, todos os serviços ofertados que recebem valores do SUS para arcarem com os gastos.

Partindo dessa explanação, este trabalho aponta a seguinte questão de investigação: Qual a viabilidade financeira entre os custos de Tomografias Computadorizadas, obtidos pelo Centro de Diagnóstico de Tratamento e o repasse monetário feito pelo SUS? Intentando responder à questão problemática desta pesquisa, propõe-se como objetivo geral, analisar a viabilidade financeira na prestação do serviço do Sistema Único de Saúde - SUS na realização de tomografias computadorizadas por uma clínica particular, situada no Alto Oeste Potiguar. Para isso, pretende-se identificar os procedimentos de tomografias computadorizadas cobertos pelo SUS; mensurar os custos operacionais na realização dos procedimentos referentes às tomografias computadorizadas cobertas pelo SUS; aferir o valor pago pelo SUS para os custos operacionais na realização dos procedimentos de tomografia computadorizadas; e verificar a viabilidade financeira de se utilizar o valor monetário recebido pelo SUS para realização dos procedimentos das tomografias computadorizadas.

Esta pesquisa poderá ser explorada para estudos futuros, nos vários níveis da instituição, inclusive pode auxiliar nos processos de tomada de decisões, dentro da organização que serviu como objeto de estudo. Também poderá ser usado de base para estudos realizados em outras organizações de outro ramo.

\title{
2. REFERENCIAL TEÓRICO
}

O referencial teórico aborda sobre a Contabilidade de Custos, a Gestão de Custos (utilizando-se do custeio $\mathrm{ABC}$ ), a formação do preço do serviço/produto e a legislação do SUS.

\subsection{Contabilidade de Custos}

Faz-se importante ressaltar que a contabilidade de custos auxilia a tomada de decisões e redução de custos operacionais e administrativos, se necessário, de modo assertivo e racional.

Leone (2010, p. 05 e 06) esclarece:

\begin{abstract}
A contabilidade de custos é o ramo da contabilidade que se destina a produzir informações para os diversos níveis gerenciais de uma entidade, como auxilio as funções de determinação de desempenho, de planejamento e controle das operações e de tomada de decisões. A contabilidade de custos coleta classifica e registra os dados operacionais das diversas atividades da entidade, denominados de dados internos, bem como, algumas vezes, coleta e organiza dados externos.
\end{abstract}

Através do que explana Martins (2009), compreende-se que a contabilidade de custos auxilia expressivamente a tomada de decisões, com ênfase no controle por intermédio dos dados fornecidos com a finalidade de estabelecer padrões e orçamentos, bem como compara valores anteriormente definidos. 
Segundo Leone (2000), a contabilidade de custos é uma atividade que objetiva o recebimento de dados, acumulando-os organizadamente para que sejam analisados e interpretados. As informações obtidas através desse processo devem ser utilizadas nos diversos níveis gerenciais. Ainda de acordo com Leone (2000), a contabilidade de custos é um conjunto de procedimentos cuja finalidade é atribuir os custos ocorridos nas atividades que procedem à fabricação e venda de um produto, auxiliando o planejamento e a mensuração de desempenho. Tais procedimentos contribuem para a gestão de custos nas organizações.

\subsection{Gestão de Custos}

Um dos maiores problemas enfrentados pelas microempresas é a realização do controle financeiro de forma eficiente e correta. Em sua grande maioria, essas organizações estão fadadas a falta de informações fidedignas sobre seus custos operacionais e administrativos, mantendo, com isso, uma administração financeira simplista utilizando métodos intuitivos.

Para que uma organização possa melhor compreender seus resultados financeiros, bem como seus preços e se for preciso tiver que realizar redução de custos de modo eficaz, faz- se necessário o mínimo de conhecimento possível sobre seus custos, obtidos desde o planejamento para a execução do serviço, ou fabricação do produto, ou da comercialização da mercadoria que influenciarão na formação do preço do serviço/mercadoria/produto.

\subsection{Formação do Preço do Serviço/Mercadoria/Produto}

Os custos possuem uma pluralidade vasta quanto a sua classificação, podem ser compreendidos em dois tipos, de acordo com a sua apropriação aos produtos fabricados, sendo os diretos, que são identificados diretamente a um produto/mercadoria/serviço, e os indiretos, que somente serão atribuídos a produtos/mercadorias/serviços por algum critério de rateio.

O presente estudo delineou ratear as duas tipologias de custos, envolvidos na realização das tomografias computadorizadas ofertadas aos usuários SUS em uma clinica privada, no interior potiguar. Utilizando o método de custeio ABC - Activity- Basead Costing, ou seja, custos baseados em atividades, averiguando os custos exatos do procedimento confrontando ao pagamento dos serviços prestados.

Segundo Martins (2009) a ferramenta ABC possibilita a identificação dos custos através das atividades executadas, diretamente e indiretamente relacionadas à fabricação dos produtos/serviços da organização. A utilização dessa ferramenta requer a definição clara dessas atividades.

De acordo com Ribeiro (2009) “Atividade é um conjunto de tarefas decorrentes da combinação de recursos humanos, financeiros, materiais e tecnológicos, que visa à produção de bens ou prestação de serviços."

Ocorre na produção desses bens ou prestações de serviços um ciclo evidenciado como forma de continuidade a realização de processos, transcrito de forma clara e simples por Ribeiro (2009) "as atividades consomem recursos e os produtos consomem atividades."

\subsection{Legislação do SUS}

O SUS, objetiva assegurar aos brasileiros, seus direitos a saúde, expressos no artigo 196 da Constituição Federal do ano de 1988 (CF/88), que enfatiza o dever do Estado para com essa obrigatoriedade. Entretanto, a administração pública, em suas modalidades: municipal, 
estadual e federal não disponibiliza de todos os serviços e especialidades médicas, através da rede pública de assistencialismo a saúde.

Sendo assim, torna-se necessário recorrer à participação das instituições privadas, que irão complementar os serviços e procedimentos a serem ofertados à sociedade, mediante várias premissas, incluindo documento de contratação, especificação e quantitativo dos serviços/procedimentos a serem ofertados, expressos em linhas gerais nos artigos 199 e 200 da $\mathrm{CF} / 88$.

Em paralelo a essa necessidade do Estado, encontram-se as microempresas, principalmente, situadas nos interiores, que buscam serem prestadores do SUS a fim de complementar seu desenvolvimento organizacional, assegurados pela alta demanda existente e consequentemente a certeza dos repasses financeiros efetuados mensalmente pelo Governo Federal, de acordo com o faturamento apresentado.

No entanto, essa terceirização de serviços/procedimentos pode não se tratar de algo tão vantajoso para os prestadores, no que diz respeito ao valor dos custos operacionais da empresa e ao valor determinado nos editais de licitações, pré-estabelecidos pelo sistema SUS, valores esses que se encontram congelados há treze anos, em contrapartida as entidades privadas sofrem os aumentos inflacionários a cada período e cada reajuste efetuado pela politica monetária nacional.

Azevedo (2016) afirma que há mais de uma década o repasse realizado torna-se insuficiente para cobrir os custos hospitalares, além do enorme descompasso no reajuste das tabelas de preços do Sistema.

\section{A TESSITURA METODOLÓGICA DA PESQUISA}

Uma pesquisa representa, sempre, a narrativa de uma longa viagem em que o pesquisador vasculha lugares, na maioria das vezes, já visitados, contudo com um modo diferente de olhar, pensar e apreender determinada realidade em função de uma experiência e de uma apropriação do conhecimento que são suscitadores de indagações, de curiosidades epistemológicas e de empreendimentos que possibilitem a construção de aquisição de informações e dados sobre um determinado objeto refletido, criticado. Afinal, o conhecimento pode ser motivado pela necessidade de conhecer algo ou saber sobre ele e, ainda, como afirma Barros; Lehfeld (2000) "É um processo de reflexão crítica cujo objetivo é o desvelamento de um objeto. "É nessa tessitura em que a prática da reflexão e da descoberta norteiam a pesquisa, a busca do conhecimento, problematizando-se o mundo vivido, sua relação com o meio e com os semelhantes e, por conseguinte, construindo representações da realidade, de forma que o sujeito possa se situar e agir no e com o mundo.

É nesse intento que a busca de entendimento acerca da dinâmica organizacional das empresas e sua saúde financeira constituem-se desafio mobilizador dessa pesquisa, a qual, em seu processo constitutivo, se realiza processualmente, a partir de três fases distintas, porém, interdependentes, perspectivando-se o desenvolvimento adequado de uma pesquisa científica que, conforme define Oliveira (2002) é um trabalho em que "É necessário planejamento cuidadoso e investigação de acordo com as normas da metodologia científica, tanto aquela referente à forma quanto a que se refere ao conteúdo."

A primeira fase resultou de uma pesquisa de campo, classificando-se a pesquisa quanto à natureza em aplicada. Nesse percurso, da investigação se materializou o contato direto com o universo da pesquisa que, conforme Minayo (2010) transfigura-se possível firmar o contato com os sujeitos/objetos de investigação, para que se possa melhor compreender e familiarizar as questões levantadas a respeito do objeto de estudo. Do ponto de vista de seus objetivos, classifica-se como exploratória, que, de acordo com Gil (2002) visa a 
aprimorar ideias ou descobrir intuições, além de apresentar um planejamento flexível, envolvendo, em geral, levantamento bibliográfico, entrevistas com pessoas que tiveram experiências práticas com o problema pesquisado e análise de exemplos similares.

$\mathrm{Na}$ segunda fase, vivenciou-se a pesquisa bibliográfica com vistas a construção de embasamento teórico acerca da temática em estudo e, mais particularmente do método de custeio $\mathrm{ABC}$, utilizado para mensuração dos custos aferidos no decorrer das atividades existentes no ambiente estudado. A pesquisa bibliográfica, do ponto de vista dos procedimentos técnicos, segundo Gil (2006).

É elaborada a partir de materiais que não receberam tratamento analítico, documentos de primeira mão, como documentos oficiais, reportagens de jornal, cartas, contratos, diários, filmes, fotografias, gravações, etc., ou, ainda, a partir de documentos de segunda mão que, de alguma forma, já foram analisados, tais como: relatórios de pesquisa, relatórios de empresas, tabelas estatísticas, etc.

Posteriormente, realizou-se a pesquisa documental que, segundo Lakatos (2003), esse método caracteriza-se por utilizar-se apenas de fontes documentais, redigidas ou não, para a obtenção de dados. Mediante a análise de relatórios internos, emitidos através dos softweres X- clínic e Quarck-clínic, que são sistemas de gestão hospitalar, pagos para manter o controle interno dos dados, sequencialmente o primeiro foi substituído pelo segundo, durante o período de realização da pesquisa, utilizou-se, também, dados registrados no software Excel 2010, cujos dados foram sistematizados e organizados à luz do referencial teórico estudado.

A terceira fase incorreu através de uma entrevista não estruturada proposta por Silva e Menezes (2001) onde a busca por informações se deu através de um roteiro flexível possibilitando discutir, amplamente, algumas questões, nesse caso, junto ao profissional responsável pela realização dos exames, sendo esse possuidor dos conhecimentos técnicos a transmitir clareza no que tange aos procedimentos operacionais, singularidades das situações diárias e suas patologias.

Compreende-se que este estudo do ponto de vista da forma de abordagem do problema caracteriza-se em qualitativo com viés quantitativo, ao que se refere a tradução dos resultados obtidos correspondente ao primeiro semestre de 2019. Em se tratando da primeira abordagem, há que se considerar que há uma relação dinâmica entre o mundo real e o sujeito, isto é, um vínculo indissociável entre o mundo objetivo e a subjetividade do sujeito que não pode ser traduzido em números. A interpretação dos fenômenos e a atribuição de significados são básicas no processo de pesquisa qualitativa. Não requer o uso de métodos e técnicas estatísticas. O ambiente natural é a fonte direta para coleta de dados, e o pesquisador é o instrumento-chave (Gil, 2006). A pesquisa qualitativa utiliza várias técnicas de dados, como a observação participante, história ou relato de vida, entrevista e outros Collis; Hussey (2005).

Ao que trata a segunda abordagem, compreende-se que tudo possa ser contável, o que significa traduzir em números opiniões e informações para classificá-las e analisá-las. Requer o uso de recursos e de técnicas estatísticas (percentagem, média, moda, mediana, desvio padrão, coeficiente de correlação e outros). Gil (2006). Assim, a pesquisa quantitativa é focada na mensuração de fenômenos, envolvendo a coleta e análise de dados numéricos e aplicação de testes estatísticos. Collis; Hussey (2005).

Os procedimentos de tomografias computadorizadas e os valores cobertos pelo SUS, foram identificados através do Sistema de Gerenciamento da Tabela de Procedimentos, Medicamentos e OPM do SUS- SIGTAP e através do contrato de licitação assinado entre a empresa e a Prefeitura Municipal da cidade em que está situada a clínica em estudo.

Quanto aos cálculos auferidos dos custos unitários da tomografia computadorizada realizada com os pacientes que são atendidos pelo SUS realizaram-se as seguintes etapas: 
$1^{\mathrm{a}}$ - Identificação das atividades realizadas;

$2^{\mathrm{a}}$ - Identificação dos recursos utilizados nas atividades;

$3^{\text {a }}$ - Identificação do consumo desses recursos;

$4^{\mathrm{a}}$ - Identificação do valor financeiro aferido a cada um dos recursos.

Em seguida, para verificar a viabilidade financeira do repasse monetário feito pelo SUS para atendimento dos pacientes, foi feito a comparação dos gastos auferidos na realização de cada tomografia computadorizada com os valores repassados pelo SUS a clinica que realiza os referidos serviços.

\section{RESULTADOS E DISCUSSÕES}

Foram elaborados dois esquemas básicos, caracterizando os setores e as atividades desenvolvidas na efetuação dos serviços prestados, o primeiro processo, compreende desde o atendimento ao cliente, na recepção, até a realização do exame, na sala de tomografia, e a liberação do paciente, e o segundo fluxograma ilustra a rotina realizada mensalmente, pretendendo com isto, demonstrar as atividades ocorridas em detrimento da prestação de contas junto à entidade competente a executar o repasse financeiro do SUS. Conforme ilustram as figuras $1(\mathrm{Um})$ e 2 (Dois).

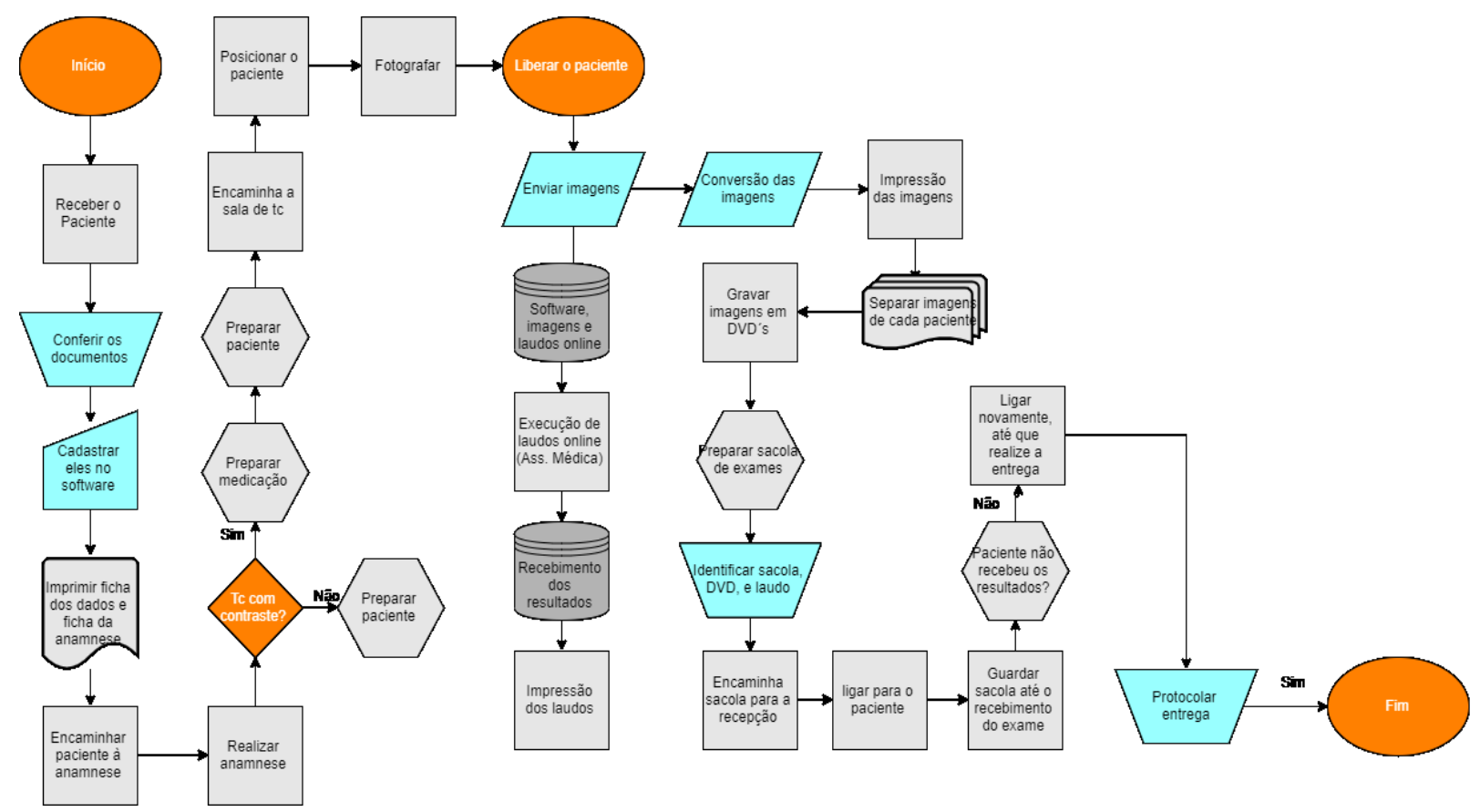

Figura 01. Percurso dos pacientes para a realização da tomografia computadorizada Fonte: Elaborada pelos autores.

Alicerçado com visualização deste fluxograma, foi possível desempenhar as etapas que decorrem no perpassar do estudo, em concordância ao método utilizado neste estudo. Por conseguinte, foram identificados os setores necessários à execução das tomografias computadorizadas, sendo eles: o setor técnico e de apoio, como também, foram identificados os setores que dão o suporte necessário para que a empresa receba os repasses do SUS, sendo o setor de faturamento. 


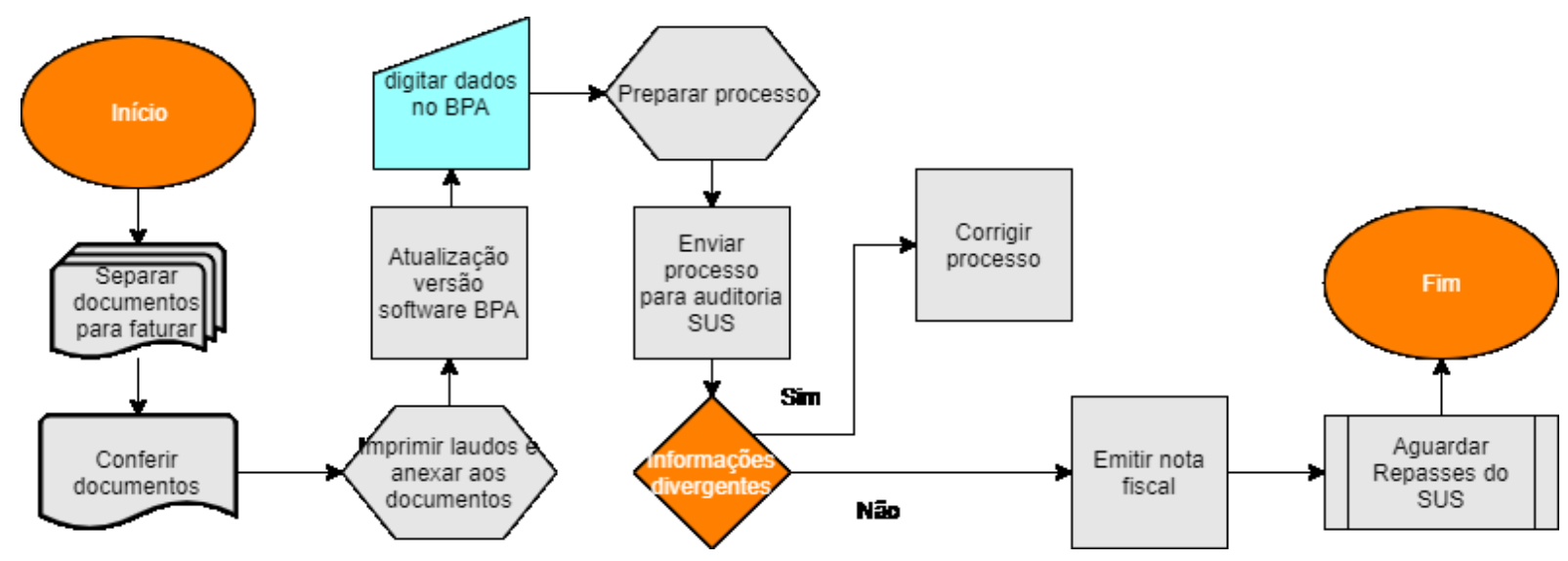

Figura 02. Atividades desenvolvidas no setor de faturamento. Fonte: Elaborada pelos autores.

Ao observar a figura 1 (um), compreende-se notoriamente que os pacientes ao serem atendidos no setor da recepção, são encaminhados à enfermeira, que irá realizar uma pequena entrevista a fim de identificar problemas de saúde que venham influenciar na execução do procedimento ou do diagnóstico. Neste interim, os mesmos deverão ser orientados a usar a vestimenta fornecida pela clínica. Em caso de exames contrastados, o paciente receberá informações, sobre como proceder, de acordo com o tipo de tomografia computadorizada (tc) solicitada pelo médico. Bem como irá ser preparado, de acordo com as práticas de enfermagem, para que seja injetado o iodo venoso.

Após todo preparo, o paciente passa a ser atendido pelo técnico em radiologia, que irá posicioná-lo da forma correta na mesa do tomógrafo e fotografá-lo no console, captando as imagens que o aparelho emite através do gantry, parte do aparelho que contém os tubos de raio-X.

Concluído a realização dos procedimentos de imagens de alta complexidade, os profissionais técnicos, liberam o paciente e sequenciam o processo, realizando ajustes na angulação das imagens, selecionando as mais importantes, para que as mesmas sejam enviadas aos médicos radiologistas, possibilitando a realização do estudo e emissão do laudo médico.

Após a entrega dos resultados, que acontecerá em até cinco dias úteis o setor do faturamento realiza a preparação do processo, que passará por uma auditoria municipal, a fim de averiguar as irregularidades e confirmação da prestação do serviço em questão, para que mediante isto, sejam solicitados os repasses financeiros do Governo Federal.

Para melhor compreensão do que foi dito, a figura 03, identifica os grupos de atividades realizadas em todo o processo.

\begin{tabular}{ll}
\hline \multicolumn{1}{c}{ GETOR DE APOIO } & \multicolumn{1}{c}{ GRUPO DE ATIVIDADES } \\
\hline \hline \multicolumn{1}{c}{ SETOR TÉCNICO } \\
I. RECEPÇÃO & I. ANAMNESE \\
II. ENTREGA DOS RESULTADOS & II. PREPARAÇÃO \\
III. FATURAMENTO & III. REALIZAÇÃO DO EXAME \\
& IV. MANUSEIO DE DADOS INFORMATIZADO \\
& V. PREPARAÇÃO DE DOCUMENTOS MANUALMENTE \\
\hline
\end{tabular}

Figura 03. Identificação das atividades

Fonte: Elaborada pelos autores. 
Finalizado a coleta de dados em campo, deu-se inicio a pesquisa documental, com a finalidade de apurar o histórico dos custos referentes ao consumo dos recursos materiais, humanos e financeiros. A primeira deu-se por identificar a quantidade de procedimentos realizados durante o período estudado, aos quais, de acordo com registros, consta que foram realizados o total de 3069 (três mil e sessenta e nove) tomografias computadorizadas, dentre as quais 1520 (um mil, quinhentos e vinte), foram procedimentos atendidos através do convênio SUS, 495 (quatrocentos e noventa e cinco) foram exames realizados através do convênio particular e 821 (oitocentos e vinte e um) foram procedimentos atendidos por intermédio de outros convênios. A figura 04 (quatro) enfatiza o comportamento da demanda durante o período estudado.

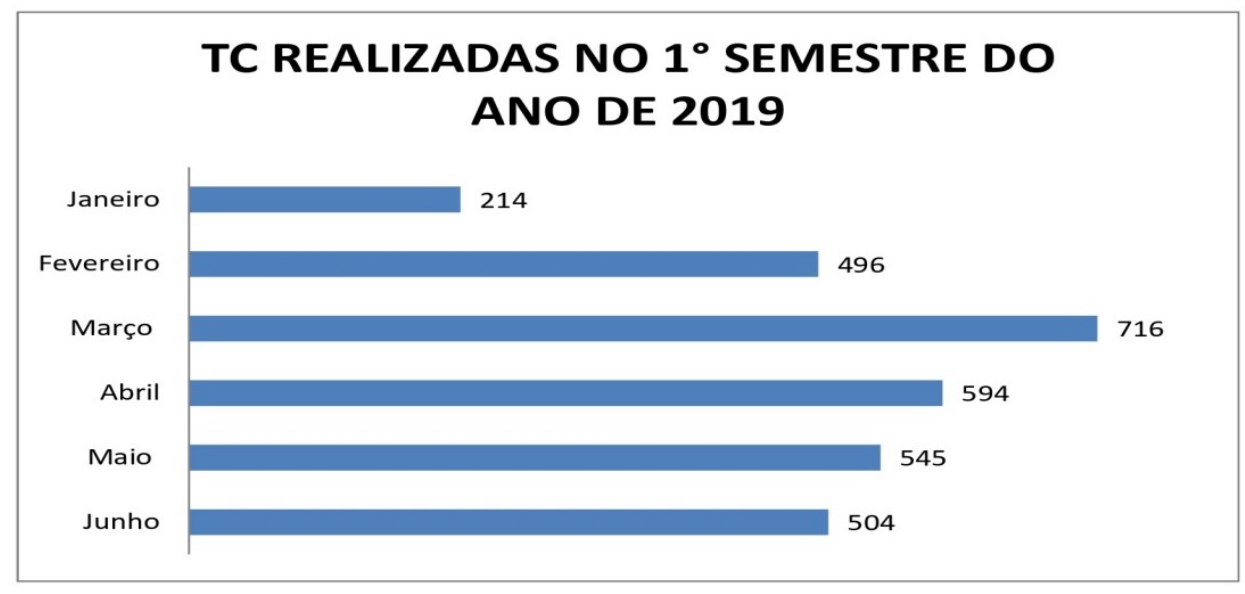

Figura 04. Comportamento da demanda Fonte: Dados da pesquisa.

Faz-se importante destacar que os primeiros 15 dias do mês de janeiro, não foram realizados nenhum tipo de procedimento, em detrimento da instalação do novo tomógrafo, dado que o aparelho antes utilizado se encontrava totalmente obsoleto e sem qualidade de uso, comprometendo a reputação da empresa em questão. Entretanto, o objetivo principal da coleta desses dados se faz pela necessidade em aferir os custos totais existentes, como será exemplificado nos gráficos a seguir.

Após ratear a quantidade total de procedimentos realizados, o presente estudo aferiu o consumo total dos recursos utilizados em todas as atividades executadas, durante o primeiro semestre do ano de 2019, calculando a média entre a quantidade de exames realizados e aferindo a média sob o valor total pago pelos recursos utilizados, durante o período em análise.

Devido às limitações encontradas para calcular o consumo de alguns recursos separados por setor e consequentemente por atividades, o estudo apresenta três tabelas a identificar os recursos aferidos por setor.

A tabela $n^{\circ} 1$ (um) identificando o consumo dos recursos comum a todas as atividades, tendo em vista que a clínica, atualmente realiza, apenas, procedimentos de tomografias computadorizadas e tendo em vista que os consultórios são restritos às consultas médicas, estes utilizariam apenas os recursos de energia e água. Mas esses consultórios não são utilizados todos os dias, sendo assim, demandam um consumo irrisório. 
Tabela 01

Recursos comuns a todas as atividades

\begin{tabular}{llrlr}
\hline $\begin{array}{c}\text { RECURS OS COMUM A TODAS } \\
\text { AS ATIVIDADES }\end{array}$ & \multicolumn{2}{c}{$\begin{array}{c}\text { GAS TOS TOTAIS } \\
\text { EM 6 MES ES }\end{array}$} & \multicolumn{2}{c}{$\begin{array}{c}\text { CUS TOS } \\
\text { MÉDIO POR TC }\end{array}$} \\
\hline \hline AGUA E SANEAM ENTO BASICO & $\mathrm{R} \$$ & 873,78 & $\mathrm{R} \$$ & 0,28 \\
AGUA POTA VEL & $\mathrm{R} \$$ & 655,00 & $\mathrm{R} \$$ & 0,21 \\
ASSISTENCIA CONTABIL & $\mathrm{R} \$$ & $5.724,00$ & $\mathrm{R} \$$ & 1,87 \\
EMPRESA DE SEGURANÇA PRIVAI & $\mathrm{R} \$$ & $1.500,00$ & $\mathrm{R} \$$ & 0,49 \\
ENERGIA & $\mathrm{R} \$$ & $16.045,85$ & $\mathrm{R} \$$ & 5,23 \\
INTERNET & $\mathrm{R} \$$ & $2.145,67$ & $\mathrm{R} \$$ & 0,70 \\
MATERIAL DE LIMPEZA & $\mathrm{R} \$$ & $1.626,57$ & $\mathrm{R} \$$ & 0,53 \\
RECURSOS HUM ANOS & $\mathrm{R} \$$ & $15.068,79$ & $\mathrm{R} \$$ & 4,91 \\
TEC. SEGURANÇA DO TRABALHO & $\mathrm{R} \$$ & $2.400,00$ & $\mathrm{R} \$$ & 0,78 \\
TELEFONE FIXO & $\mathrm{R} \$$ & $1.217,88$ & $\mathrm{R} \$$ & 0,40 \\
TELEFONE MOVEL & $\mathrm{R} \$$ & 807,94 & $\mathrm{R} \$$ & 0,26 \\
\hline FOn
\end{tabular}

Fonte: Dados da pesquisa.

O custo total de cada item foi dividido pela quantidade de tomografias computadorizadas realizadas na clínica durante período analisado.

Nota-se que nesta tabela a energia e os recursos humanos são os mais onerosos e que a água potável é o recurso menos oneroso.

A tabela $\mathrm{n}^{\circ} 2$ (dois) ilustra os recursos utilizados apenas nas atividades realizadas no setor de apoio.

Tabela 02

Recursos inerentes ao setor de apoio

\begin{tabular}{|l|rrr|r}
$\begin{array}{c}\text { RECURS OS UTILIZADOS SOMENTE NAS } \\
\text { ATIVIDADES DO S ETOR DE APOIO }\end{array}$ & \multicolumn{2}{c|}{$\begin{array}{c}\text { GAS TOS TOTAIS } \\
\text { EM 6 MES ES }\end{array}$} & $\begin{array}{c}\text { CUS TOS MÉDIO } \\
\text { POR TC }\end{array}$ \\
\hline \hline DEPRECIAÇÃO DA IMPRESSORA & $\mathrm{R} \$$ & 30,69 & $\mathrm{R} \$$ & 0,01 \\
DEPRECIAÇÃO DO AR CONDICIONADO & $\mathrm{R} \$$ & 398,97 & $\mathrm{R} \$$ & 0,13 \\
DEPRECIAÇÃO DOS COMPUTADORES & $\mathrm{R} \$$ & 245,52 & $\mathrm{R} \$$ & 0,08 \\
IMPLANTAÇÃO SIST.QUARCK & $\mathrm{R} \$$ & 259,80 & $\mathrm{R} \$$ & 0,48 \\
MATERIAIS DE EXPEDIENTE & $\mathrm{R} \$$ & $1.012,77$ & $\mathrm{R} \$$ & 0,33 \\
RECURSOS HUM ANOS & $\mathrm{R} \$$ & $11.969,10$ & $\mathrm{R} \$$ & 3,90 \\
SISTEMA RECEPÇÃO E FINANCEIRO & $\mathrm{R} \$$ & 368,28 & $\mathrm{R} \$$ & 0,12 \\
\hline
\end{tabular}

Fonte: Elaborada pelos autores.

Percebem-se com a tabela apresentada que o recurso mais oneroso e o menos oneros são respectivamente os recursos humanos e a depreciação do equipamento de impressão. A depreciação foi calculada utilizando o valor de mercado, em virtude da dificuldade de encontrar as notas fiscais de compra dos equipamentos. $\mathrm{O}$ cálculo obedeceu à determinação dada pela Secretaria da Receita Federal, no artigo 305 do RIR/99, que estipula o prazo de 10 anos para depreciação de máquinas e equipamentos. Desse modo, compreende-se que a taxa de depreciação anual se dá em $10 \%$ sob o valor total do bem, consequentemente será de $5 \%$ ao semestre, o valor obtido através do cálculo de depreciação foi dividido pela quantidade de exames realizados nos seis primeiros meses objetivando identificar o valor da depreciação atribuída a cada procedimento já realizado.

A tabela 03 (três), por sua vez, exibe o consumo dos recursos inerentes às atividades realizadas no setor técnico. 


\begin{tabular}{|c|c|c|}
\hline \multirow{2}{*}{$\begin{array}{l}\text { RECURSOS UTILIZADOS SOMENTE NAS ATIVIDADES DO SETOR TÉCNICO } \\
\text { APOLICE DE SEGUROS DO TOMOGRAFO }\end{array}$} & \multicolumn{2}{|c|}{ CUSTOS MÉDIO POR TC } \\
\hline & $\mathrm{R} \$$ & 0,35 \\
\hline CERTIFICADO CONS. REG. MEDICINA & $\mathrm{R} \$$ & 0,25 \\
\hline COLETA LIXO HOSPITALAR & $\mathrm{R} \$$ & 0,28 \\
\hline CONS. REG. TEC. RAD. & $\mathrm{R} \$$ & 0,01 \\
\hline CRM DIRETOR TÉCNICO & $\mathrm{R} \$$ & 0,12 \\
\hline DEPRECIAÇÃO DO AR CONDICIONADO & $\mathrm{R} \$$ & 0,10 \\
\hline DEPRECIAÇÃO DO TOMOGRAFO & $\mathrm{R} \$$ & 12,22 \\
\hline DVD PARA IMAGENS & $\mathrm{R} \$$ & 0,80 \\
\hline LAUDO MÉDICCO & $\mathrm{R} \$$ & 35,00 \\
\hline LEASING IMPRESSORA DE IMAGENS & $\mathrm{R} \$$ & 3,17 \\
\hline LEV. RADIOMETRICO & $\mathrm{R} \$$ & 0,32 \\
\hline LICENÇA SANITÁRIA & $\mathrm{R} \$$ & 0,15 \\
\hline MANUTENÇÃO AR CONDICIONADO & $\mathrm{R} \$$ & 0,09 \\
\hline MATERIAL HOSPITALAR & $\mathrm{R} \$$ & 0,11 \\
\hline MEDIÇÃO DE DOSES RADIOMETRICAS & $\mathrm{R} \$$ & 0,21 \\
\hline RECURSOS HUMANOS & $\mathrm{R} \$$ & 12,60 \\
\hline SACOLA PARA ENTREGA DO EXAME & $\mathrm{R} \$$ & 3,00 \\
\hline SIS. ON LINE DE LAUDOS/ ARQUNV EM NUVEM & $\mathrm{R} \$$ & 3,61 \\
\hline SISTEMA DE CONV IMAGENS & $\mathrm{R} \$$ & 1,63 \\
\hline TESTE DE QUALIDADE DO EQUIPAMENTO & $\mathrm{R} \$$ & 0,10 \\
\hline
\end{tabular}

Fonte: Dados da pesquisa.

Para calcular a depreciação dos equipamentos, como ar condicionado, utilizou-se do valor de mercado, respeitando as características a que possuem como marca e modelo, pois uma das principais dificuldades da pesquisa foi encontrar as notas fiscais de compra dos equipamentos.

A depreciação do tomógrafo foi calculada através da nota fiscal de compra, tendo em vista que o mesmo foi instalado no mês de janeiro de 2019.

Os cálculos do consumo das folhas de impressão das imagens foram realizados a partir de uma entrevista totalmente aberta junto ao supervisor técnico em radiologia. Ao qual o mesmo quantificou o número das folhas utilizadas na impressão das imagens de cada tipo de procedimento, como mostra a tabela de $n^{\circ} 4$ (quatro).

De acordo com a fala "a quantidade de folhas utilizadas podem variar de acordo, com os tipos de patologias existentes, em cada paciente e isso é algo muito difícil de quantificar, entende? Porque quando a gente está realizando o exame na hora, a gente vê alguma coisa, que precisa ser fotografada, que ajuda na interpretação da imagem, para que o médico laude, depende também da indicação médica, o que o médico prescreve na solicitação do exame. Então a gente faz o que vai garantir a qualidade de um bom exame. Não dá para ter isso em uma tabela. Mas em casos clínicos, em condições normais, esses gastos, apontados na tabela 04 corresponde às quantidades de folhas por exame". 
Tabela 04

Consumo de papel couche para impressão de imagens

\begin{tabular}{lccccc}
\hline \multicolumn{1}{c}{$\begin{array}{c}\text { Tipos de Tomografia } \\
\text { Computadorizada }\end{array}$} & $\begin{array}{c}\mathbf{N}^{\circ} \text { de } \\
\text { Folhas }\end{array}$ & Valor unt & Valor total \\
\hline \hline Abdome sup erior & 3 & $\mathrm{R} \$$ & 0,60 & $\mathrm{R} \$$ & 1,80 \\
Articulações Sacróiliacas & 3 & $\mathrm{R} \$$ & 0,60 & $\mathrm{R} \$$ & 1,80 \\
Bacia & 3 & $\mathrm{R} \$$ & 0,60 & $\mathrm{R} \$$ & 1,80 \\
Coluna cervical & 3 & $\mathrm{R} \$$ & 0,60 & $\mathrm{R} \$$ & 1,80 \\
Coluna lombar & 3 & $\mathrm{R} \$$ & 0,60 & $\mathrm{R} \$$ & 1,80 \\
Coluna toracica & 3 & $\mathrm{R} \$$ & 0,60 & $\mathrm{R} \$$ & 1,80 \\
Cotovelo & 4 & $\mathrm{R} \$$ & 0,60 & $\mathrm{R} \$$ & 2,40 \\
Coxa & 5 & $\mathrm{R} \$$ & 0,60 & $\mathrm{R} \$$ & 3,00 \\
Crânio & 3 & $\mathrm{R} \$$ & 0,60 & $\mathrm{R} \$$ & 1,80 \\
Face & 3 & $\mathrm{R} \$$ & 0,60 & $\mathrm{R} \$$ & 1,80 \\
Joelho & 5 & $\mathrm{R} \$$ & 0,60 & $\mathrm{R} \$$ & 3,00 \\
Mastóide & 3 & $\mathrm{R} \$$ & 0,60 & $\mathrm{R} \$$ & 1,80 \\
Ombro & 4 & $\mathrm{R} \$$ & 0,60 & $\mathrm{R} \$$ & 2,40 \\
Pé & 4 & $\mathrm{R} \$$ & 0,60 & $\mathrm{R} \$$ & 2,40 \\
Pelve & 3 & $\mathrm{R} \$$ & 0,60 & $\mathrm{R} \$$ & 1,80 \\
Perna & 5 & $\mathrm{R} \$$ & 0,60 & $\mathrm{R} \$$ & 3,00 \\
PescoçO & 4 & $\mathrm{R} \$$ & 0,60 & $\mathrm{R} \$$ & 2,40 \\
Seios da Face & 2 & $\mathrm{R} \$$ & 0,60 & $\mathrm{R} \$$ & 1,20 \\
Sela turcica & 3 & $\mathrm{R} \$$ & 0,60 & $\mathrm{R} \$$ & 1,80 \\
Toráx & 2 & $\mathrm{R} \$$ & 0,60 & $\mathrm{R} \$$ & 1,20 \\
Tornozelo & 4 & $\mathrm{R} \$$ & 0,60 & $\mathrm{R} \$$ & 2,40 \\
\hline Font Da & & & & & \\
& & & &
\end{tabular}

Fonte: Dados da pesquisa.

O desgaste do tubo de raio $\mathrm{x}$, também foi calculado com a ajuda do entrevistado onde em sua fala esclarece: "é meio complicado quantificar o uso do tubo, porque depende de muitas coisas, dos cortes axiais, de variáveis subjetivas, que diz respeito a forma de uso, como está sendo utilizado a máquina, quantas vezes foi preciso aquecer o tubo, os exames foram feitos só pela manhã, ou a tarde também, tinha muito exame naquele dia, ou fez um exame passou um tempo, o tubo esfriou, chegou outro. Está me entendendo? É como falei a respeito das folhas, dar para calcular em condições normais, mas depende das patologias, do problema de saúde do paciente, depende da espessura do corte, e da indicação do médico, se o médico pedir um exame conjugado com outro, é muito relativo, todo dia temos uma situação nova. "

Após a entrevista, realizou-se a simulação de compra de um tubo, onde se obteve as seguintes informações, o valor de um tubo de raio-x para ser utilizado no modelo e marca do aparelho de tomografia instalado no ambiente de pesquisa, custa $\mathrm{R} \$ 127.994,70$, com garantia de 1 (um) ano ou 100.000 (cem mil) cortes axiais, o que primeiro ocorrer, muito embora o tubo possa realizar até 200.000 (duzentos mil) cortes, o fabricante, confirma durante a simulação de compra que essa duração depende da forma de uso, da demanda existente no local. Por conveniência, estimou-se o valor dos cortes, dividindo o valor de compra pela quantidade de cortes cobertos pela garantia do fabricante.

Cálculo realizado para quantificar o valor de um corte axial.

$$
\mathrm{R} \$ 127.994,70 / 100.000=\mathrm{R} \$ 1,27
$$

Desse modo, a quantidade de cortes foi quantificada para cada tipo de procedimento realizado em condições patológicas dentro dos padrões da normalidade, com auxilio das imagens projetadas no monitor do console e análise do supervisor em radiologia, que foi identificando quais exames estavam dentro do padrão "normal". A tabela no 05 (cinco) registra os dados coletados quanto aos cortes axiais e suas espessuras. Ressalva a espessura do 
corte axial, que se dá em milímetros, e que segundo o técnico entrevistado "quanto mais fino o corte, mais haverá desgaste do tubo de raio - x ".

Tabela 05

Consumo e espessuras dos cortes axiais.

\begin{tabular}{|c|c|c|c|c|c|c|}
\hline $\begin{array}{l}\text { Tipos de Tomografia } \\
\text { Computadorizada }\end{array}$ & $\begin{array}{l}N^{\circ} \text { de cortes } \\
\text { axiais }\end{array}$ & mm do corte & & lor & Val & or total \\
\hline Abdome superior & 50 & $5,0 \mathrm{~mm}$ & $\mathrm{R} \$$ & 1,27 & $\mathrm{R} \$$ & 63,50 \\
\hline Articulações Sacroíliacas & 38 & $3,75 \mathrm{~mm}$ & $\mathrm{R} \$$ & 1,27 & $\mathrm{R} \$$ & 48,26 \\
\hline Bacia & 50 & $5,0 \mathrm{~mm}$ & R\$ & 1,27 & $\mathrm{R} \$$ & 63,50 \\
\hline Coluna cervical & 32 & $5,0 \mathrm{~mm}$ & $\mathrm{R} \$$ & 1,27 & $\mathrm{R} \$$ & 40,64 \\
\hline Coluna lombar & 43 & $5,0 \mathrm{~mm}$ & $\mathrm{R} \$$ & 1,27 & $\mathrm{R} \$$ & 54,61 \\
\hline Coluna toracica & 75 & $5,0 \mathrm{~mm}$ & $\mathrm{R} \$$ & 1,27 & $\mathrm{R} \$$ & 95,25 \\
\hline Cotovelo & 50 & $3,75 \mathrm{~mm}$ & $\mathrm{R} \$$ & 1,27 & $\mathrm{R} \$$ & 63,50 \\
\hline Coxa & 90 & $5,0 \mathrm{~mm}$ & $\mathrm{R} \$$ & 1,27 & $\mathrm{R} \$$ & 114,30 \\
\hline Crânio & 35 & $5,0 \mathrm{~mm}$ & R\$ & 1,27 & $\mathrm{R} \$$ & 44,45 \\
\hline Face & 40 & $5,0 \mathrm{~mm}$ & $\mathrm{R} \$$ & 1,27 & $\mathrm{R} \$$ & 50,80 \\
\hline Joelho & 50 & $3,75 \mathrm{~mm}$ & $\mathrm{R} \$$ & 1,27 & $\mathrm{R} \$$ & 63,50 \\
\hline M astóide & 191 & $0,62 \mathrm{~mm}$ & $\mathrm{R} \$$ & 1,27 & $\mathrm{R} \$$ & 242,57 \\
\hline Ombro & 50 & $5,0 \mathrm{~mm}$ & $\mathrm{R} \$$ & 1,27 & $\mathrm{R} \$$ & 63,50 \\
\hline Pé & 50 & $3,75 \mathrm{~mm}$ & $\mathrm{R} \$$ & 1,27 & $\mathrm{R} \$$ & 63,50 \\
\hline Pelve & 50 & $5,0 \mathrm{~mm}$ & R\$ & 1,27 & $\mathrm{R} \$$ & 63,50 \\
\hline Perna & 117 & $3,75 \mathrm{~mm}$ & $\mathrm{R} \$$ & 1,27 & $\mathrm{R} \$$ & 148,59 \\
\hline Pescoço & 68 & $3,75 \mathrm{~mm}$ & $\mathrm{R} \$$ & 1,27 & $\mathrm{R} \$$ & 86,36 \\
\hline Seios da Face & 33 & $3,75 \mathrm{~mm}$ & $\mathrm{R} \$$ & 1,27 & $\mathrm{R} \$$ & 41,91 \\
\hline Sela turcica & 20 & $3,75 \mathrm{~mm}$ & $\mathrm{R} \$$ & 1,27 & $\mathrm{R} \$$ & 25,40 \\
\hline Toráx & 60 & $5,0 \mathrm{~mm}$ & $\mathrm{R} \$$ & 1,27 & $\mathrm{R} \$$ & 76,20 \\
\hline Tornozelo & 50 & $3,75 \mathrm{~mm}$ & $\mathrm{R} \$$ & 1,27 & $\mathrm{R} \$$ & 63,50 \\
\hline
\end{tabular}

Fonte: Dados da pesquisa.

Através da entrevista também foi possível identificar os procedimentos que são realizados com a aplicação do contraste venoso, ainda, sem a utilização da bomba injetora.

A pesquisa documental e de campo identificaram que os exames contrastados também são ofertados através do SUS, muito embora, não exista nenhum repasse financeiro a mais para suprir os custos aferidos a esses procedimentos. Em conformidade com as tabelas informadas, no Sistema de Gerenciamento da Tabela de Procedimentos, Medicamentos e OPM do SUS-SIGTAP, o procedimento pode ser contrastado ou não. Diante disso, aferiu-se os custos sob os 4 (quatro) tipos de procedimentos contrastados, mais solicitados pelo SUS. A ser identificados na tabela 06 (seis).

Tabela 06

Tomografias computadorizadas com contraste

\begin{tabular}{lccccccccccc}
\hline $\begin{array}{c}\text { Tipos de TC com } \\
\text { contraste }\end{array}$ & $\begin{array}{c}\mathbf{N}^{\mathbf{0}} \text { de } \\
\text { Folhas }\end{array}$ & Valor unt & Valor total & $\begin{array}{c}\text { Quantidade } \\
\text { de cortes }\end{array}$ & mm & valor unt & Valor total \\
\hline \hline Abdome superior & 6 & $\mathrm{R} \$$ & 0,60 & $\mathrm{R} \$$ & 3,60 & 100 & $5 \mathrm{~mm}$ & $\mathrm{R} \$ 1,27$ & $\mathrm{R} \$$ & 127,00 \\
Crânio & 6 & $\mathrm{R} \$$ & 0,60 & $\mathrm{R} \$$ & 3,60 & 70 & $5 \mathrm{~mm}$ & $\mathrm{R} \$ 1,27$ & $\mathrm{R} \$$ & 88,90 \\
Pelve & 6 & $\mathrm{R} \$$ & 0,60 & $\mathrm{R} \$$ & 3,60 & 100 & $5 \mathrm{~mm}$ & $\mathrm{R} \$ 1,27$ & $\mathrm{R} \$ 127,00$ \\
Toráx & 4 & $\mathrm{R} \$$ & 0,60 & $\mathrm{R} \$$ & 2,40 & 120 & $5 \mathrm{~mm}$ & $\mathrm{R} \$ 1,27$ & $\mathrm{R} \$ 152,40$ \\
\hline
\end{tabular}

Fonte: Dados da pesquisa.

Conferem-se, conjuntamente, na tabela 07 (Sete), os custos dos materiais utilizados a aplicação do iodo venoso ou oral. 
Tabela 07

Materiais hospitalares

\begin{tabular}{|ccccr}
\hline \multicolumn{5}{c|}{ Materias utilizados } \\
\hline \hline Qtd & und de medida & Descrição & \multicolumn{2}{c|}{ Valor } \\
\hline \hline 1 & und de medida & catete jelco & $\mathrm{R} \$$ & 2,85 \\
100 & $\mathrm{ml}$ & omnip aque ioexol & $\mathrm{R} \$$ & 100,00 \\
5 & und & seringas & $\mathrm{R} \$$ & 4,50 \\
1 & und & dispositivo 02 vias & $\mathrm{R} \$$ & 1,50 \\
1 & und & agulha p aspiração & $\mathrm{R} \$$ & 0,25 \\
1 & und & band aid & $\mathrm{R} \$$ & 0,25 \\
1 & $\mathrm{~g}$ & algodão & $\mathrm{R} \$$ & 0,02 \\
10 & cm & esparadrapos & $\mathrm{R} \$$ & 0,10 \\
2 & und & luvas & $\mathrm{R} \$$ & 0,35 \\
\hline
\end{tabular}

Fonte: Elaborada pelos autores.

Através das tabelas anteriores, foi possível mensurar o custo total para cada tipo de procedimento ofertado e compará-los ao valor pago pelo SUS. Desta forma, a tabela 08 (Oito), enfatiza a comparação dos valores.

Tabela 08

Comparativo entre custos reais $\mathrm{x}$ valores pagos pelo SUS.

\begin{tabular}{|c|c|c|c|c|c|c|c|}
\hline $\begin{array}{l}\text { Codigo } \\
\text { S IGTAP }\end{array}$ & $\begin{array}{l}\text { Tipos de Tomografia } \\
\text { Computadorizada }\end{array}$ & \multicolumn{2}{|c|}{ Valor S US } & \multicolumn{2}{|c|}{$\begin{array}{c}\text { Custo total } \\
\text { unt. }\end{array}$} & \multicolumn{2}{|c|}{ Déficit } \\
\hline $02.06 .03 .001-0$ & Abdome superior & $\mathrm{R} \$$ & 138,63 & $\mathrm{R} \$$ & 160,13 & $-\mathrm{R} \$$ & 21,50 \\
\hline $02.06 .03 .002-9$ & Articulações Sacroíliacas & $\mathrm{R} \$$ & 86,75 & $\mathrm{R} \$$ & 137,27 & $-\mathrm{R} \$$ & 50,52 \\
\hline $02.06 .03 .003-7$ & Bacia & $\mathrm{R} \$$ & 138,63 & $\mathrm{R} \$$ & 151,24 & $-\mathrm{R} \$$ & 12,61 \\
\hline $02.06 .01 .001-0$ & Coluna cervical & $\mathrm{R} \$$ & 86,76 & $\mathrm{R} \$$ & 191,88 & $-\mathrm{R} \$$ & 105,12 \\
\hline $02.06 .01 .002-8$ & Coluna lombar & $\mathrm{R} \$$ & 101,10 & $\mathrm{R} \$$ & 160,13 & $-\mathrm{R} \$$ & 59,03 \\
\hline $02.06 .01 .003-6$ & Coluna toracica & $\mathrm{R} \$$ & 86,76 & $\mathrm{R} \$$ & 210,93 & $-\mathrm{R} \$$ & 124,17 \\
\hline $02.06 .02 .001-5$ & Cotovelo & $\mathrm{R} \$$ & 86,75 & $\mathrm{R} \$$ & 141,68 & $-\mathrm{R} \$$ & 54,93 \\
\hline $02.06 .02 .002-3$ & Coxa & $\mathrm{R} \$$ & 86,75 & $\mathrm{R} \$$ & 148,63 & $-\mathrm{R} \$$ & 61,88 \\
\hline $02.06 .01 .007-9$ & Crânio & $\mathrm{R} \$$ & 97,44 & $\mathrm{R} \$$ & 160,13 & $-\mathrm{R} \$$ & 62,69 \\
\hline $02.06 .01 .004-4$ & Face & $\mathrm{R} \$$ & 86,75 & $\mathrm{R} \$$ & 339,20 & $-\mathrm{R} \$$ & 252,45 \\
\hline $02.06 .03 .002-9$ & Joelho & $\mathrm{R} \$$ & 86,75 & $\mathrm{R} \$$ & 161,33 & $-\mathrm{R} \$$ & 74,58 \\
\hline $02.06 .01 .007-9$ & Mastóide & $\mathrm{R} \$$ & 97,44 & $\mathrm{R} \$$ & 160,13 & $-\mathrm{R} \$$ & 62,69 \\
\hline $02.06 .02 .001-5$ & Ombro & $\mathrm{R} \$$ & 86,75 & $\mathrm{R} \$$ & 160,73 & $-\mathrm{R} \$$ & 73,98 \\
\hline $02.06 .02 .002-3$ & Pé & $\mathrm{R} \$$ & 86,75 & $\mathrm{R} \$$ & 245,82 & $-\mathrm{R} \$$ & 159,07 \\
\hline $02.06 .03 .003-7$ & Pelve & $\mathrm{R} \$$ & 138,63 & $\mathrm{R} \$$ & 182,99 & $-\mathrm{R} \$$ & 44,36 \\
\hline $02.06 .02 .002-3$ & Perna & $\mathrm{R} \$$ & 86,75 & $\mathrm{R} \$$ & 139,74 & $-\mathrm{R} \$$ & 52,99 \\
\hline $02.06 .01 .005-2$ & Pescoço & $\mathrm{R} \$$ & 86,75 & $\mathrm{R} \$$ & 122,63 & $-\mathrm{R} \$$ & 35,88 \\
\hline $02.06 .01 .004-4$ & Seios da Face & $\mathrm{R} \$$ & 86,75 & $\mathrm{R} \$$ & 172,23 & $-\mathrm{R} \$$ & 85,48 \\
\hline $02.06 .01 .006-0$ & Sela turcica & $\mathrm{R} \$$ & 97,44 & $\mathrm{R} \$$ & 122,03 & $-\mathrm{R} \$$ & 24,59 \\
\hline $02.06 .02 . .003-1$ & Toráx & $\mathrm{R} \$$ & 136,41 & $\mathrm{R} \$$ & 172,23 & $-\mathrm{R} \$$ & 35,82 \\
\hline $02.06 .03 .002-9$ & Tornozelo & $\mathrm{R} \$$ & 86,75 & $\mathrm{R} \$$ & 160,73 & $-\mathrm{R} \$$ & 73,98 \\
\hline $\begin{array}{l}\text { Codigo } \\
\text { S IGTAP } \\
\end{array}$ & Tipos de $T C$ com contraste & \multicolumn{2}{|c|}{ Valor S US } & \multicolumn{2}{|c|}{$\begin{array}{c}\text { Custo total } \\
\text { unt. } \\
\end{array}$} & \multicolumn{2}{|c|}{ Déficit } \\
\hline $02.06 .03 .001-0$ & Abdome superior & $\mathrm{R} \$$ & 138,63 & $\mathrm{R} \$$ & 225,43 & $-\mathrm{R} \$$ & 86,80 \\
\hline $02.06 .01 .007-9$ & Crânio & $\mathrm{R} \$$ & 97,44 & $\mathrm{R} \$$ & 187,33 & $-\mathrm{R} \$$ & 89,89 \\
\hline $02.06 .03 .003-7$ & Pelve & $\mathrm{R} \$$ & 138,63 & $\mathrm{R} \$$ & 225,43 & $-\mathrm{R} \$$ & 86,80 \\
\hline $02.06 .02 . .003-1$ & Toráx & $\mathrm{R} \$$ & 136,41 & $\mathrm{R} \$$ & 248,63 & $-\mathrm{R} \$$ & 112,22 \\
\hline
\end{tabular}

Fonte: Dados da pesquisa.

A tabela acima identifica cada tipo de tomografia computadorizada, realizada na referida clinica através do convênio SUS, elencando a receita obtida por cada tipo de procedimento e comparando ao valor do custo unitário, calculado através do método de 
custeio $\mathrm{ABC}$. Como se pode ver, o resultado dessa diferença é um saldo negativo, em todos os exames realizados.

\section{CONSIDERAÇÕES FINAIS}

Este estudo buscou ratear, através do método $\mathrm{ABC}$, os recursos humanos, materiais e financeiros, que são inerentes à realização dos exames de imagens, bem como identificou cada tipo de procedimento realizado e consequentemente construiu a mensuração dos custos por tipo de exame, objetivando compará-los a receita obtida através do condicionamento de ser prestador de serviços junto ao Governo Federal.

Através dos dados obtidos por intermédio do estudo de caso, da pesquisa documental e da entrevista realizada fica evidenciado o déficit sob o valor de cada tipo de tomografia computadorizada à ser contratado pelo SUS, deixando clara a necessidade de reaver os valores contratuais dos procedimentos estudados.

Conquanto os custos mensurados superem as receitas obtidas, recomenda-se ponderação ao decidir continuar prestando serviços ou não através do SUS. Pois o estudo também mostra que os procedimentos realizados pelo convênio SUS representam $49,5 \%$ da realização total de exames, enquanto que o convênio particular demandou apenas 16,1\%, bem como os outros tipos de convênios demandaram a soma de $34,4 \%$.

A pesquisa realizada possui várias limitações, no tocante, houve dificuldades em encontrar documentos que haviam sido desprezados, não foi possível mensurar o desgaste do tubo de raio-x ao que diz respeito a forma de uso.

For fim, recomenda-se que estudos futuros sejam realizados sob o intuito de averiguar os aspectos positivos e negativos que a demanda SUS pode causar a organização, partindo da premissa de que as receitas são deficitárias a manutenção dos custos.

\section{REFERÊNCIAS}

Azevedo, Y. G. P.; Santos, J. V. J. G.; Gomes, A. M.; Carvalho, D. R.; \& Oliveira, R. M. A. (2016). Análise de custeio em exames de diagnóstico por imagem: Um estudo de caso utilizando o método activity- based costing. Rev. Eletrônica Gestão \& Saúde, 7 (3), p. 1106-1125.

Barros, A. J. S.;\& Lehfeld, N.A.S. (2000). Fundamentos de metodologia científica: um guia para a iniciação científica. (2a ed. rev., e ampl.). São Paulo: Makron Books.

Bedê, M. A. (2016). Sobrevivência das empresas no Brasill. Brasília: SEBRAE.

BRASIL. Constituição (1988). Constituição da República Federativa do Brasil. Texto constitucional promulgado em 5 de outubro de 1988, Disponível em: $<$ http://www.planalto.gov.br/ccivil_03/constituicao/constituicao.htm $>$. Acesso em: 20 Julho 2019.

Collis, J.; \& Hussey, R. (2005). Pesquisa em Administração: um guia prático para alunos de graduação e pós-graduação. (2a. ed.). Porto Alegre: Bookman.

Gil, A. C. (2002). Como elaborar projetos de pesquisa. (4a. ed.). São Paulo: Atlas.

Gil, A. C. (2006). Métodos e técnicas de pesquisa social. (5a.ed.). São Paulo: Atlas.

Amaro Júnior, E.; \& Yamashita, H. (2001). Aspectos básicos de tomografia computadorizada e ressonância magnética. Rev. Bras. Psiquiatría, 23 (supl.1), p. 2-3.

Lakatos, E. M.; \& Marconi, M. A. (2003). Fundamentos de metodologia científica. (5a ed.). São Paulo: Atlas.

Leone, G. S. G. (2010). Curso de conatbilidade de custos. (4a ed.). São Paulo: Atlas. 
Leone, G. S. G. (2000). Custos: planejamento implantação e controle. (3a ed.) São Paulo: Atlas.

Martins, E. (2009). Contabilidade de custos. (9a ed.). São Paulo : Atlas.

Minayo, M. C. S. (2010). Pesquisa Social: Teoria, método e criatividade. (21 a ed.). Petropolis: Editora Vozes.

Oliveira, S. L. (2002). Tratado de metodologia científica: projetos de pesquisa, TGI, TCC, monografias, dissertações e teses. São Paulo: Pioneira Thomson Learning.

Ribeiro, O. M. (2009). Contabilidade de custos fácil. (7a ed. ampl. e atual.). São Paulo: Saraiva.

Silva, E. L. (2001). Metodologia da pesquisa e elaboração de Dissertação. (3a ed. rev. atual.). Florianópolis: Laboratório de Ensino a Distância da UFSC. 